\title{
Mechanical Percolation in Nanocomposites: Microstructure and Micromechanics
}

\author{
Sarah C. Baxter ${ }^{a}$, Brian J. Burrows ${ }^{b}$ and Bethany S. Fralick ${ }^{c}$ \\ ${ }^{a}$ School of Engineering, University of St. Thomas, St. Paul, MN \\ ${ }^{b}$ Department of Mechanical Engineering, University of South Carolina, Columbia, SC \\ ${ }^{c}$ Department of Mathematical Sciences, University of South Carolina-Aiken, Aiken, SC
}

\begin{abstract}
Polymer nanocomposites can enable innovative designs of multifunctional materials. Metallic fillers in polymer matrices exhibit improved electrical properties at low volume fractions, often maintaining the low density, transparency and easy processing of polymers. Surprisingly, enhanced mechanical properties have also been observed at uncharacteristically low volume fractions in these nanocomposites. The majority of mathematical models used to describe this novel mechanical behavior are based on percolation models of microstructural connectivity. Changes in mechanical properties, however, are likely to be affected by complex microstructures, beyond simply connected, as well as by the micromechanical mechanisms associated with a composite material. Both microstructural and micromechanical mechanisms are thought to be significantly influenced by the presence and properties of an interface region, between particles and matrix, which functions as a third composite phase. In this work, the relative influence of the competing and compounding effects of the spatial position/distribution of the particles (microstructure) and of the composite constitution (micromechanics) are ex-
\end{abstract}


amined. The results show that models based solely on the inclusion of a third composite phase, do not predict the experimentally observed mechanical response. This work continues with a study of the micromechanical effects of microstructure using a probabilistic and statistical characterization of the local strain fields associated with random microstructures. These continuous fields are not only more amenable to statistical characterization than the spatial ternary (matrix, particle and interface) fields that describe the microstructure, but offer a more direct, and potentially more visual, link between microstructure and mechanics. An apparent percolation threshold for a $2 \mathrm{D}$ material model is identified based on statistical characterization of the elastic moduli, distributions of local strains and spatial autocorrelation of local strain fields. The statistics of strain fields associated with microstructures producing minimum and maximum moduli are also compared.

Keywords: A. Nanocomposites, B. Mechanical properties B. Interface C. Probabilistic methods

\section{Introduction}

Enhanced mechanical properties have been observed in polymer nanocomposites at uncharacteristically low volume fractions $[1,2,3]$. These effects are thought to be due, in part, to the significant scale effect of the matrix-particle interface region in nanocomposites, $[4,5,6]$. This interface region occurs as a result of a perturbation of the properties of the matrix material due to the presence of the included particles. Factors that may cause this perturbation are, for example, the quality of bonding between the material phases, confinement of the matrix, or interference in the mobility of the flexible chains 
of the polymer. These factors can result in an increase in the stiffness of the matrix in a thin layer surrounding the reinforcing particles, effectively creating a third composite phase. These local effects, and the development of this interface region, are present in all composite materials, but because of the high surface area to volume ratio at the nanoscale, the volume fraction of the interface in nanocomposites can be greater than that of the particles, representing a significant, stiffer than the matrix, composite phase. In addition to contributing to the overall effective stiffness of the composite it has been hypothesized that these interfacial regions contribute to the formation of percolated microstructures by forming connections between particles and interface, a pseudo-percolation, [5, 7, 8, 9], or by percolating themselves.

The majority of application based models used to describe percolation effects depend on prior knowledge of a theoretical percolation threshold. This threshold is the volume fraction at which a connected microstructure within a random composite is likely to form. Models based on percolation thresholds are well developed in the study of electrically conducting composites. Because of the resemblance of the mechanical curves to electrical percolation curves, many of the electrical models have been adopted to describe mechanical effects; conductivity terms are replaced by stiffness terms. However, while composite electrical conductivity is relatively binary; below the threshold volume fraction the composite has low conductivity and above the threshold its conductivity is greatly enhanced, mechanical percolation may have more intermediate stages. Certainly a connected microstructure will enhance mechanical properties, but composite properties are continuously affected by the volume fraction of filler. Additionally, when a compliant ma- 
trix is confined between unconnected regions of a stiffer included phase, the ability of the matrix to deform may be reduced, making it effectively stiffer.

A number of researchers have attempted to include more mechanics in modeling mechanical percolation. Early work included the Generalized Effective Media model, [10], which interpolated between a mean field model, at low volume fractions, and percolation theory, above the percolation threshold. This model has been used to predict both electrical and mechanical percolation, [3]. The series-parallel model, [11], included an intermediate parameter that described the volume fraction of material that was active in the transfer of forces. A limitation of both of these models is that a previously identified value for the percolation threshold is required as input. In [12], the authors examined the influence of an interface region, as well as the effects of clustering using the concentric cylinder micromechanics model, but not in the context of percolation thresholds. A hybrid numerical analytic model was used in [13] to investigate polymer nanocomposites with complex microstructural configurations; the model included the effects of an interface as a third, independent phase, i.e. not linked to particle placement..

\section{Microstructure and Micromechanics}

Fralick et al., [9], studied percolation effects by simulating populations of random microstructures for a three phase nanocomposite, particles, matrix and interface, at discrete volume fractions. Effective properties of each microstructure were calculated and statistical averages of these properties used to predict composite response. In this approach composite mechanical response defines the percolation threshold, rather than the reverse. This 
model also predicted a distribution of properties resulting from the random microstructures; these probabilistic distributions varied with particle volume fraction [14].

The most easily identifiable microstructures, which produced significant increases in stiffness, were those where particle and interface regions formed connected pathways, a pseudo-percolation, [8]. However, these microstructures were difficult to associate with the distribution of intermediate stiffnesses observed in the population of simulated microstructures and expected as a result of the random placement of particles. This distribution of properties is likely due to a combination of mechanisms, potentially dominated by microstructure effects, spatial arrangements, percolation and/or pseudopercolation, but also affected by the micromechanical analysis that includes a third composite phase.

To investigate the relative contributions of these effects, the results presented in [9], which used the grid based computational Generalized Method of Cells (GMC) micromechanics model, [15, 16], are compared to the predictions of an analytic micromechanics model, Mori-Tanaka (MT), [17, 18], to provide an illustration of the competing and compounding effects of spatial position and composite constitution. In what follows a brief overview of both models is presented.

\subsection{GMC/Simulation Model}

In [9], the model nanocomposite was a polymer matrix embedded with metallic nanoparticles. Random microstructures were simulated at volume fractions ranging from 0 - 1 in steps of $0.05 ; 300$ microstructural realizations were generated for each volume fraction. The Generalized Method of Cells 
micromechanics model was used to calculate effective elastic properties in the simulated microstructures. GMC is a periodic unit cell model that uses a rectangular repeating unit cell (RUC), composed of multiple subcells, as the representative volume element (RVE). The nanocomposite model consisted of a cubic RUC with cubic subcells. Each subcell is assigned the specific properties of one of the phases, matrix, particles or interface. The homogenization process in GMC connects the material microstructure to an equivalent homogeneous material through a set of continuum level equations. Periodic boundary conditions are used to enforce continuity of displacements and tractions across subcell boundaries and between RUCs.

Each composite phase was assigned isotropic elastic properties. The metal particle subcells were assigned a stiffness of $E_{p} \sim 10^{10} \mathrm{~Pa}$, and a Poisson's ratio of 0.33 and the polymer matrix subcells a stiffness of $E_{m} \sim 10^{4} \mathrm{~Pa}$ and a Poisson's ratio of 0.45 . There are no accurate measurements of interfacial stiffness, however it is reasonable to expect that the interface will have a stiffness between that of the matrix and the included phase. Here the interface region was assigned a stiffness equal to the geometric mean of the polymer and particles, $\sqrt{E_{m} E_{p}}$ and a Poisson's ratio of 0.45. Interface thickness was set at one-half of the particle diameter based on an assumed interface thickness of $15 \mathrm{~nm}$, a value often mentioned in the literature, surrounding a 30 nm diameter particle.

Figure 1 shows the GMC/Simulation results, plotting the minimum, mean and maximum values of the elastic stiffness. All values are scaled by the matrix stiffness, $E_{\text {composite }} / E_{m}$. The increase in properties on the log-scale plot, between volume fractions of 0.20 and 0.35 , characterizes an 'apparent' 


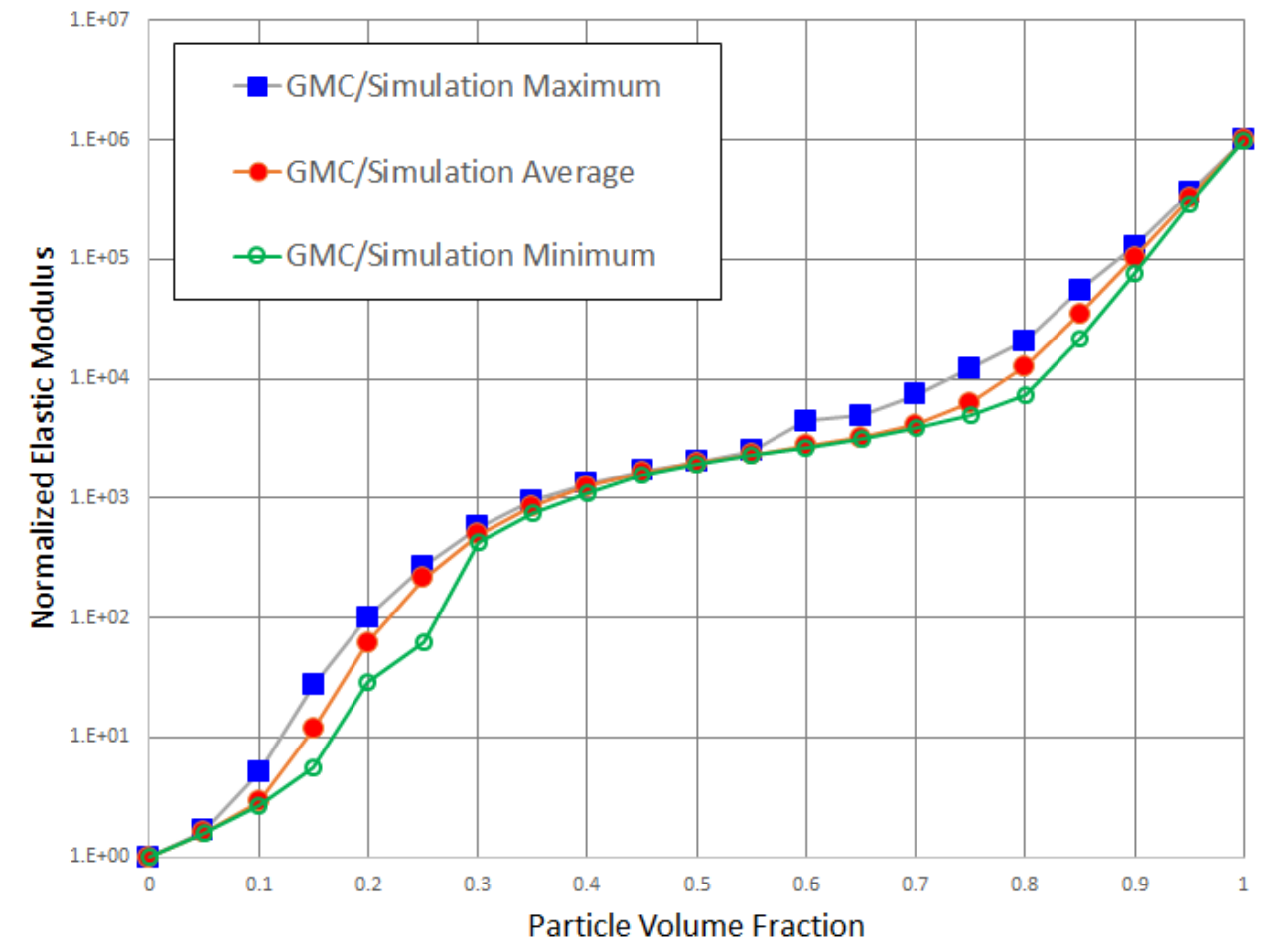

Figure 1: Scaled elastic moduli for 3-phase nanocomposite based on the GMC/Simulation model results. Minimum, mean and maximum values vs particle volume fraction. 
percolation threshold effect.

In the GMC/Simulation model the interface region was simulated as an effect of particle placement rather than as a distinct third composite phase, i.e., particle subcell positions were established first and interface subcells were inserted around them. Interface subcells were allowed to replace matrix subcells and other interface subcells, but never particle subcells. This resulted in variable interface volume fractions depending on the relative clustering of particles. Figure 2 plots the average volume fraction of each phase versus particle volume fraction. Most significant are the points at a volume fraction, $v f=0.2$, where the volume fraction of interface becomes larger than the volume fraction of matrix material; at $v f=0.35$, roughly where the volume fraction of interface reaches its maximum; and at $v f=0.45$ where the particle volume fraction is finally larger than either of the separate volume fractions of matrix or interface.

\subsection{3-Phase Mori-Tanaka Model}

The GMC/Simulation model includes the combined effects of spatial position as well as the added effect of the presence and properties of a third composite phase, the interface. While specific microstructures with connected pathways of particle and interface material, could be identified, most of the microstructures did not show obvious signs of percolation. To investigate the specific contribution of the additional stiffness of the interface to

the effective composite properties, predictions of the mechanical response of the same nanocomposite material were made using the Mori-Tanaka (MT) micromechanics model adapted for three composite phases. Mori-Tanaka is considered most accurate at low volume fractions but because it is a well- 


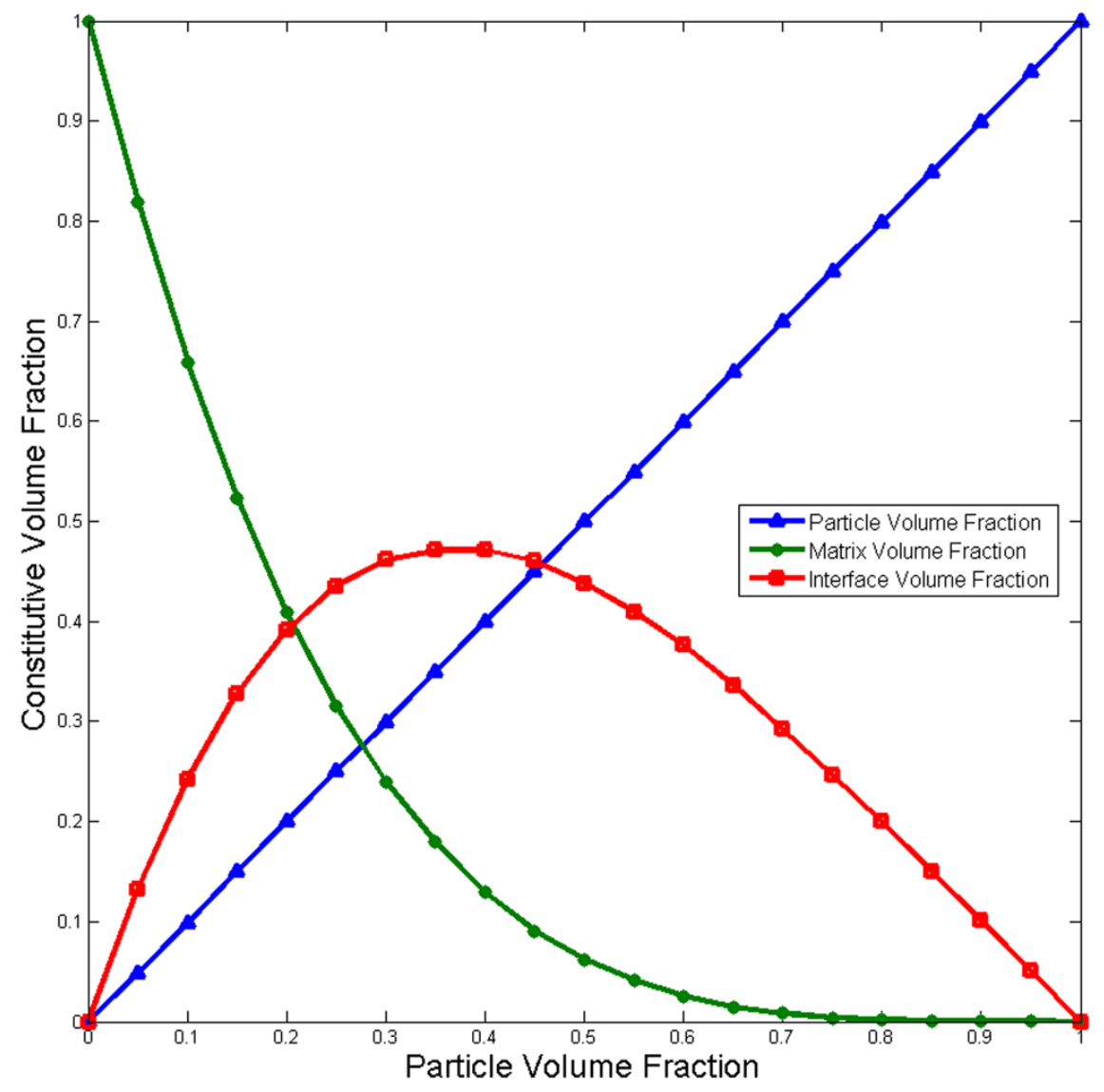

Figure 2: Phase volume fractions vs particle volume fraction. As particle volume fraction increases, interface volume fraction increases and matrix volume fraction decreases. Once the volume fraction of matrix reaches zero, interface subcells are replaced by particles subcells. 
established and frequently used model in the literature, it is used here to provide a valuable comparison over the full range. MT assumes that individual particles 'see' an effective medium around them. The properties of this effective medium depend on the presence of the other phases. As a result, although MT includes the effect of surrounding particles, considered closer or farther away, it does not reflect the direct influence of spatial position.

Based on this approach MT defines the effective composite stiffness tensor, $C^{\star}$, as

$$
C^{\star}=\left(C^{m} v_{m}+v_{i f} C^{i f} T^{i f}+v_{P} C^{p} T^{p}\right)\left(v_{m} I+v_{i f} T^{i f}+v_{p} T^{p}\right)^{-1}
$$

where $C_{i}, i=m$, if , or $p$ are the stiffness tensors associated with the matrix, interface and particle respectively. The volume fractions of each phase are similarly denoted as $v_{i}, i=m$, if , or $p$. $I$ is the identity tensor. The $T^{i}, i=$ $m$, if, or $p$, are concentration tensors, which relate local and global strains, in the individual phases. These are based on the dilute approximation, [18], and defined in terms of the $4^{\text {th }}$ order Eshelby tensor, $S^{i}$, for spherical particles, [19].

$$
\begin{aligned}
T^{p} & =\left(I+S^{p}\left(C^{m}\right)^{-1}\left(C^{m}-C^{p}\right)\right) \\
T^{i f} & =\left(I+S^{i f}\left(C^{m}\right)^{-1}\left(C^{m}-C^{p}\right)\right)
\end{aligned}
$$

The volume fractions of the individual phases used in MT were drawn from the average values calculated in the GMC/Simulation model shown in fig. 2. The results, therefore, reflect the increase and decrease of the volume fraction of the interface region.

In GMC the equations of continuous displacements and strains are enforced in an average sense, so the corners of the squares do not have a signif- 


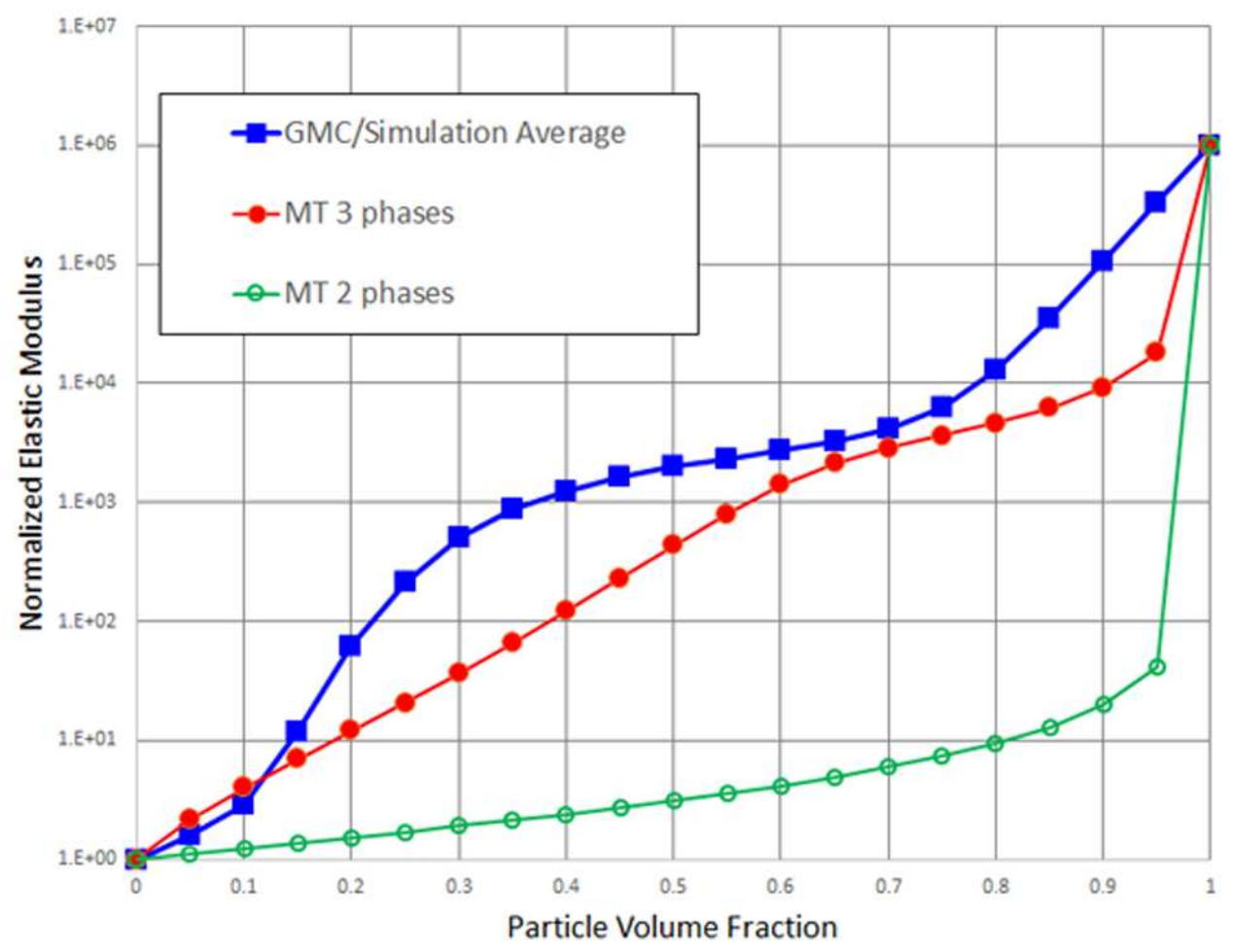

Figure 3: Based on the 3-phase nanocomposite, average properties predicted from the GMC/Simulation model are compared to properties predicted by the MT model. MT predictions for a 2-phase nanocomposite are also shown. MT predicts a significant, but gradual, increase in stiffness due to the interface. The GMC/Simulation predictions exhibit a more classic percolation type response. 
icant impact of the effective elastic properties. Because of this, the effective elastic properties of spherical particles predicted by MT can be compared to those predicted by GMC for cubic particles.

Figure 3 compares the average response using the GMC/Simulation model to the 3-phase Mori-Tanaka (MT) model. Predictions of properties for a twophase, using the MT model, are also shown for comparison.

The effect of the third phase, in the interval between $v f=[0,0.60]$, shows a linear trend in composite properties with increasing particle volume fraction. This trend mimics that of the 2-phase model; the 3-phase composite curve has a steeper slope. Between $v f=\left[\begin{array}{ll}0.60 . & 0.70\end{array}\right]$, the volume fraction of the matrix material diminishes to zero. The material is now a 2-phase composite, interface and particles, and the MT 3-phase model again follows the same trend as the two phase model trend, but at higher effective stiffnesses.

While the MT model clearly shows the significant micromechanical effect of a third composite phase, it does not capture any of the effects of irregular random microstructure., e.g. connectivity or clustering. The larger, and more abrupt, increase in stiffness predicted by the GMC/Simulation model suggests that microstructure may contribute the larger changes in magnitude of stiffness. The distribution of properties observed in the GMC/Simulation models, though, suggests that the combination of microstructure and micromechanics may be required to produce, and model, the observed percolationlike effects. 


\section{Micromechanical Effects of Microstructure}

There is a significant body of work in the literature on methods that can be used to develop statistical descriptions characterizing spatial positions in random microstructures, e.g. correlations based on the microstructure itself, [20]. However, these ternary fields are noisy, (e.g., 0, 1, 2, for particles, matrix and interface) and not directly linked to resulting mechanical properties. In this work a different approach is taken. Rather than using the microstructure itself, local strains fields based on the microstructure are examined as a possible metric for tracking microstructurally linked mechanical mechanisms. These strain fields are less noisy, they are relatively continuous fields and as a result, somewhat better data sets for statistical analysis.

Random microstructures, simulated and characterized as described above, were computationally loaded in strain. These strain fields were then characterized using probabilistic metrics and the tools of spatial statistics [21]. Probability distribution functions (PDFs) for the strains in each of the phases were constructed using the Principle of Maximum Entropy, [22, 23]. These distributions are compared within and across a range of volume fractions. In the final part of the work, autocorrelation fields are used to compare mechanical effects in minimum and maximum modulus microstructures.

\subsection{Modeling and Simulation}

Random microstructures were simulated for the same nanocomposite as described in the section above. The GMC/Simulation model was used to predict the effective composite properties. The level of detail in the threedimensional models makes visualization of field data extremely difficult, so 


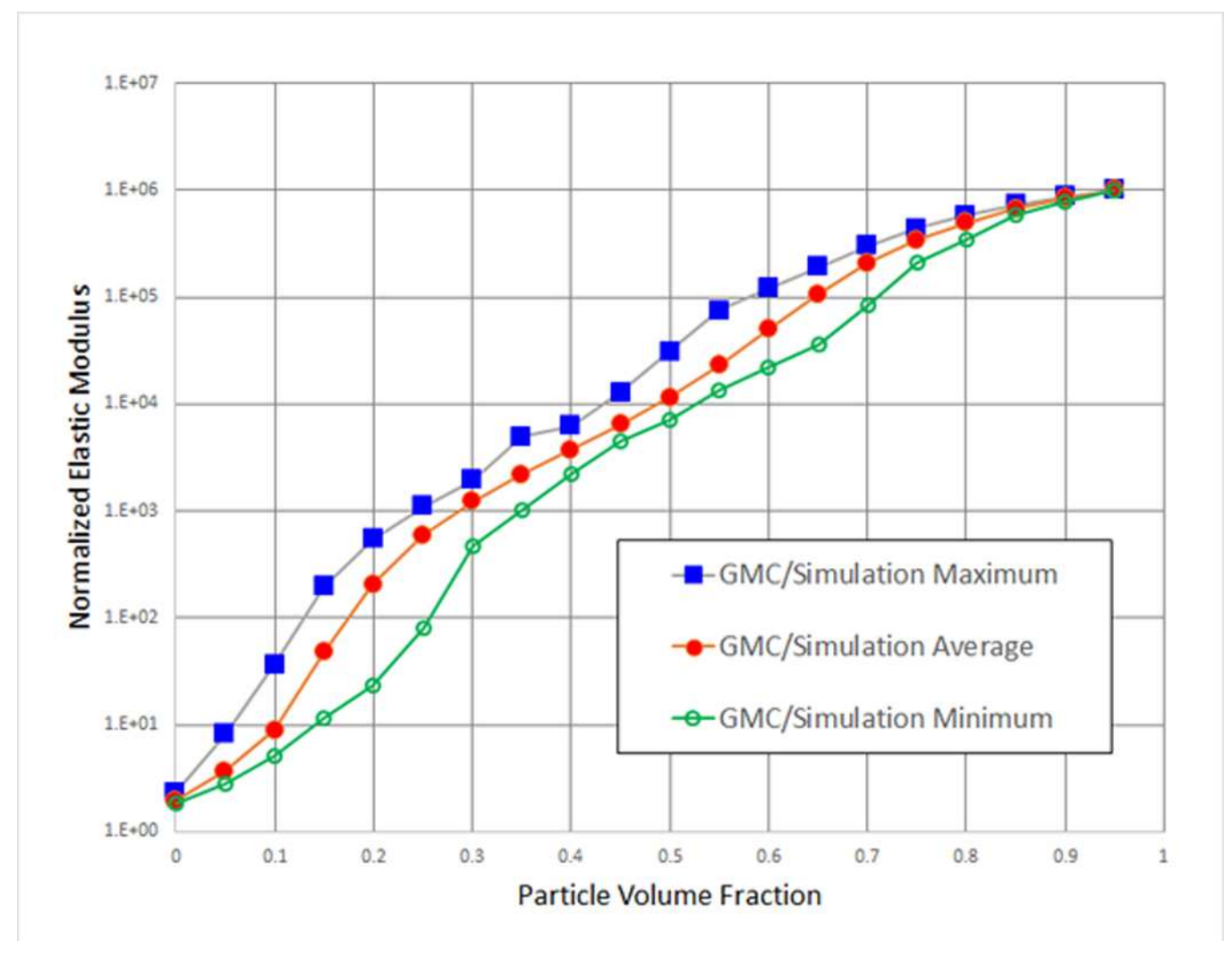

Figure 4: Scaled elastic moduli for 2-phase nanocomposite based on the GMC/Simulation model results. Minimum, mean and maximum values vs particle volume fraction. 
this initial investigation was performed on two-dimensional microstructures. High-Fidelity GMC, (HFGMC) was used for the local analysis. HFGMC is a recent formulation of GMC that provides more accurate predictions of local stress and strain fields [16]. HFGMC corresponds well to comparable FEM analyses, but is computationally efficient for simulation studies. Simulations were performed at various volume fractions in steps of $0.05 ; 300$ microstructural realizations were generated for each volume fraction.

Figure 4 shows the simulation results, plotting the minimum, mean and maximum values of the normalized elastic stiffness, $E_{\text {composite }} / E_{m}$. A comparison of the results of the three-dimensional GMC/Simulation model, fig. 1, to those of the two-dimensional GMC/Simulation model, fig. 4, illustrates the effect of the dimension of the model. The general trends, e.g., locations of inflection points are similar, but the spread over the minimum and maximum curves is wider, the curves are steeper and the plateau at mid-range is almost eliminated in the 2D model. This is most likely the effect of dimension on the scaling laws associated with random fields.

The increase in properties on the log-scale plot, between volume fractions of 0.20 and 0.35 , suggests an 'apparent' percolation threshold effect.

Figure 5 shows the skew statistics of the distribution of elastic moduli at each volume fraction. This metric can be used to refine estimates of the percolation threshold. The peak value at a volume fraction 0.15 suggests the emergence of the first microstructures that produce uncharacteristically large moduli; these microstructures are few in number and dramatically skew the distribution curves. The regions on the graph where the skew approaches zero, volume fractions $0.22-0.40$, indicate a more symmetric distribution of 


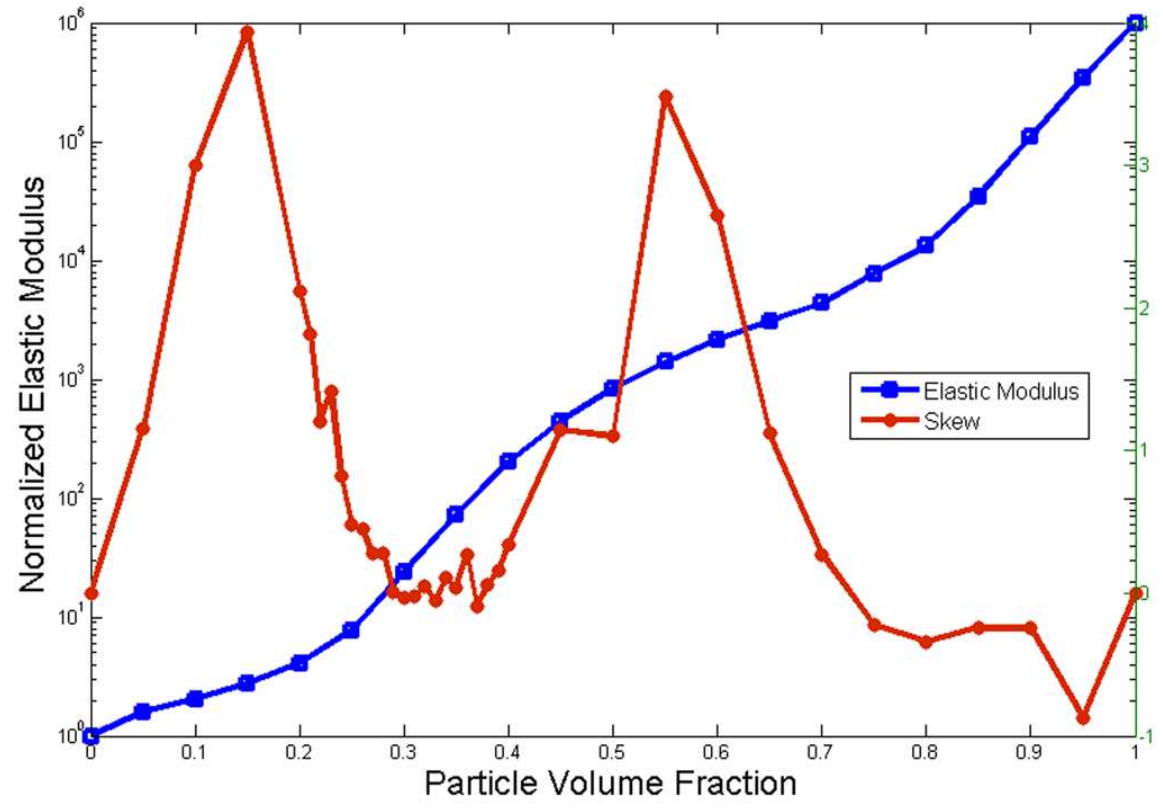

Figure 5: Skew statistics of elastic moduli at each volume fraction overlaid on modulus vs volume fraction plot. 
the moduli. It seems likely that an 'apparent' percolation threshold, where the majority of simulated microstructures result in a stiffer composite material, is within this range. Percolation thresholds, based on simulations, are traditionally defined as those volume fractions where $50 \%$ or more of the microstructures show connectivity, [24].

\subsection{Probability Density Functions}

Probability density functions of the strains in each of the three phases, particle, interface and matrix, were constructed using the Principle of Maximum Entropy [22]. The Principle of Maximum Entropy says that of all the possible solutions, the one that maximizes an entropy term, provided that one accounts for all a priori information, should be chosen. This optimization results in a choice of solution with the maximum uncertainty, or the minimum embedded bias.

Shannon's entropy functional, $H[p(E \mid v)]$, is traditionally used as the measure of uncertainty, [22], where $p(E \mid v)$ is the probability of the value of the elastic modulus, $E$, given the conditions of the composite phase properties and volume fraction, $v$. The distribution function, $p(E \mid v)$, is then defined as the function that maximizes

$$
H[p(E \mid v)]=-\int_{-\infty}^{\infty} p(E \mid v) \ln [p(E \mid v)] d E .
$$

The moduli are assumed to be bounded, with a minimum, $E^{-}$, and a maximum, $E^{+}$such that

$$
E^{-} \leq E \leq E^{+}
$$


and the mean value, $\bar{E}$ is assumed known. This information is enough to define a first-order PDF, which satisfies the conditions,

$$
\int_{-\infty}^{\infty} p(E \mid v) d E=1 \quad\left(0^{t h} \text { moment }\right)
$$

the normalization of the area under the PDF to one, and

$$
\int_{-\infty}^{\infty} p(E \mid v) E d E=\bar{E}\left(1^{s t} \text { moment }\right) .
$$

which is the mean, $\bar{E}$.

A second order PDF will satisfy the additional constraint of the $2^{\text {nd }}$ moment,

$$
\int_{-\infty}^{\infty} p(E \mid v) E^{2} d E=\text { variance }\left(2^{n d} \text { moment }\right)
$$

Higher order PDFs must satisfy conditions based on higher order moment equations, making the general form of the $N^{t h}$ order distribution function

$$
p(E \mid v)=\exp \left(\lambda_{0}+\sum_{n=1}^{N} \lambda_{n} E^{n}\right) \quad E^{-} \leq E \leq E^{+}, p(E \mid v)=0, \text { otherwise }
$$

where the Lagrange multipliers, the $\lambda$ 's, are solved for based on the imposed constraints. A detailed description of this formulation can be found in [25].

The variational problem defined by eqn (4), subject to the constraints stated in eqns. (5), (6), (7) and (8), based on the sampled distribution of strains in the matrix and interface regions, was solved for second order PDFs. The strain values were drawn from microstructures with the maximum moduli at particle volume fractions of $0.22,0.25,0.28,0.31$ and 0.34 . These 
0.22
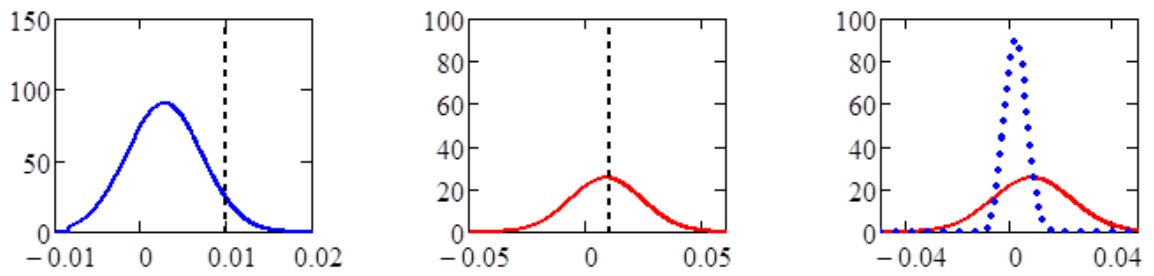

0.25
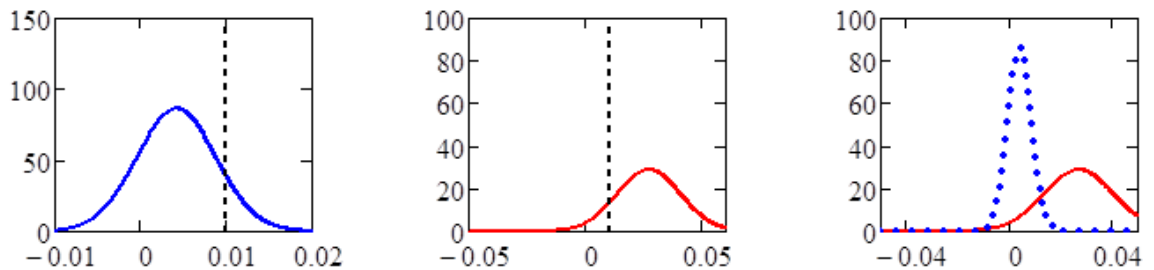

0.28
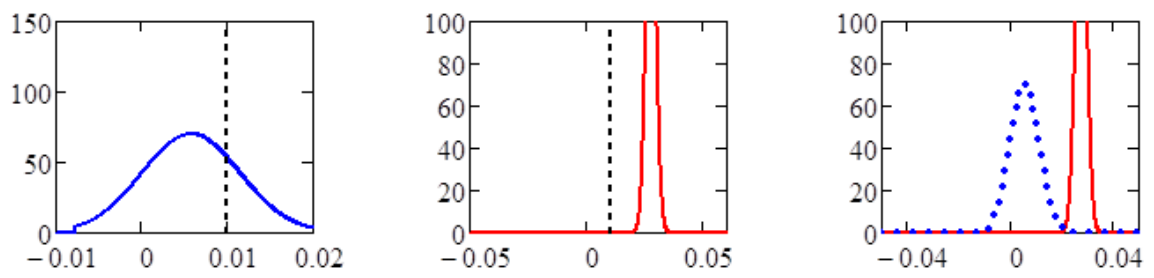

0.31
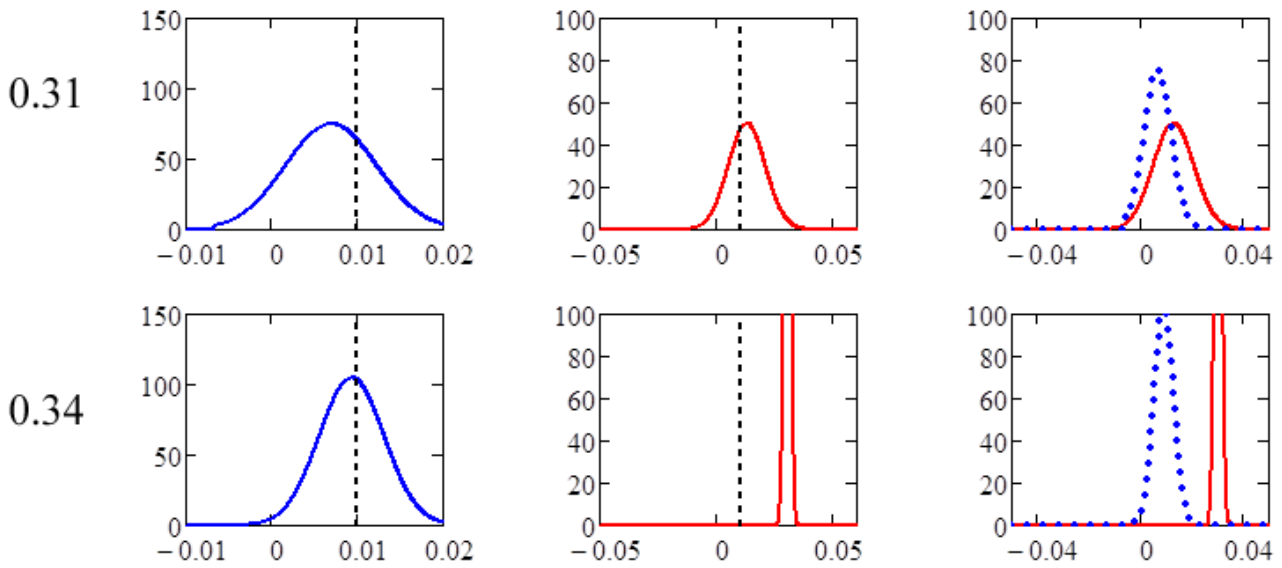

Interface Max

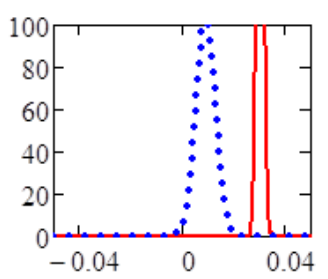

Interface/Matrix

Figure 6: PDFs of strains in interface and matrix subcells; maximum modulus microstructures. Dotted lines in first two columns represent the uniform applied strain, $\epsilon^{0}$. Dotted line in the third column is interface strain PDF, solid line is matrix PDF. 
PDFs are shown in Figure 6. Strains in the particle subcells were distributed over extremely small ranges and resulted in narrow, almost single valued distribution functions; they were not plotted.

The distributions of strains in the interface regions show that the range of deformation is small, consistent with constraints due to the attachment to the stiff particle phase. Over these volume fractions, the most probable strain value in the interface increased from below the applied strain, $\epsilon^{0}$, to approximately equal to it. In this range, volume fractions of interface increase with particle volume fraction and the interface represents the largest component of the composite.

Distributions of strains in the matrix show a wider range of values, and less consistent trends. Figure 4 suggests that at volume fractions of 0.22 and 0.25 , regions of matrix are mostly in tension, but not uniformly constrained by any reinforcement due to the microstructure. At a volume fraction of 0.28 , the strains are more narrow suggesting a more homogeneous microstructure. At 0.31, while the distribution of strains widens, it also shifts to lower values, again suggesting some reinforcing effect of the stiffer phases. The shift to higher strains again at 0.34 may be the result of the significantly lower volume fraction of matrix.

The distribution of strains in microstructures with minimum and maximum moduli, interface and matrix subcells, respectively, are compared in Figure 5. Mean strains in the interface regions of the maximum modulus microstructures were generally higher than in the minimum microstructures. In contrast, mean strains in the matrix regions of the maximum modulus microstructures were roughly equal to those in the minimum microstructures, 
but the distribution in the minimums was consistently wider. Again, a significant change in the distributions occurs at a particle volume fraction of 0.28 .

Mean strains in the particle phase, (PDFs not shown in figure), although small, were consistently higher in the maximum modulus microstructures than minimum, on the order of $10^{-6}$ versus $10^{-7}$ at 0.22 and 0.25 and on the order of $10^{-5}$ versus $10^{-7}$ at the higher volume fractions.

\section{Autocorrelation}

Spatial autocorrelation is the average covariance over all pairs of observations whose locations differ by a vector $(k, l)[21]$. In this work the autocorrelation function in MatLab, xcorr2, $\AA$, was used to generate spatial statistical plots. In the original formulation of two-dimensional autocorrelation of an $M$ by $N$ matrix, $X$ with itself, is defined as the matrix array $C$,

$$
\begin{aligned}
C(k, l)= & \Sigma_{m=0}^{M-1} \Sigma_{n=0}^{N-1} X(m, n) \bar{X}(m-k, n-l) \\
& -(M-1) \leq k \leq M-1 \\
& -(N-1) \leq l \leq N-1
\end{aligned}
$$

where the overbar denotes complex conjugation. In order to reduce the boundary effects, a doubly periodic array, $3 \times 3$ of the original microstructure is correlated with a single version of the microstructure. Final images were then cropped from the larger array output. 
0.22
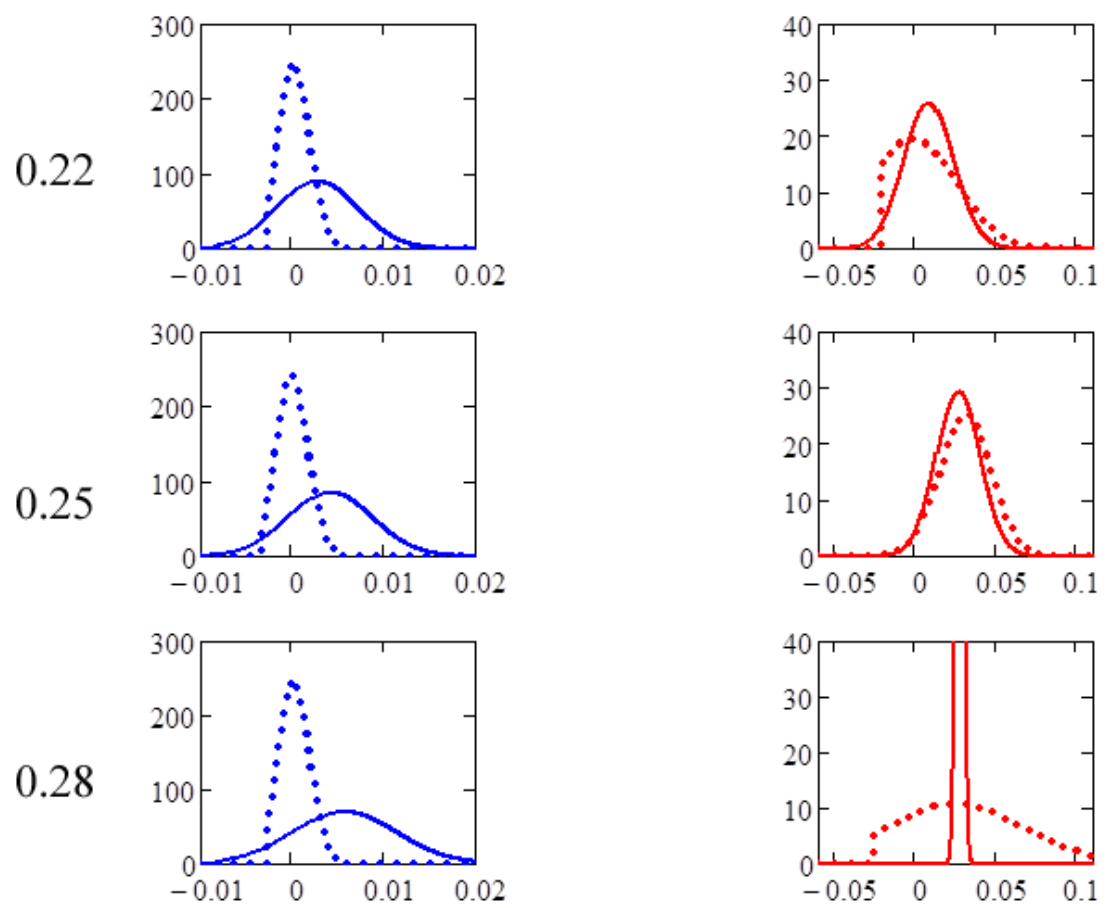

0.31
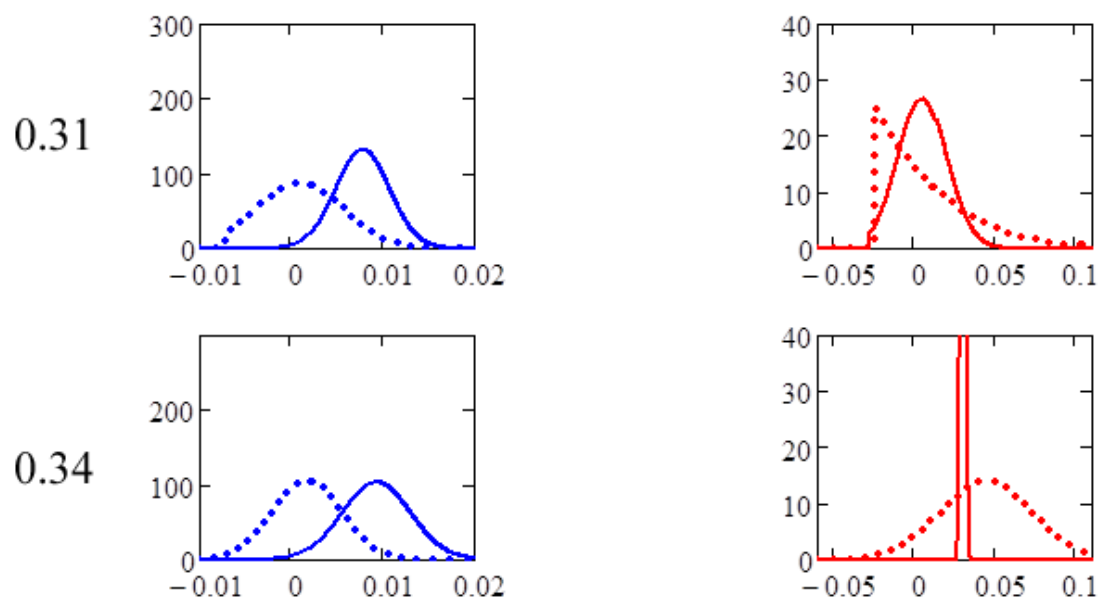

Interface Min/Max

\section{Matrix Min/Max}

Figure 7: Distributions of strains in interface and matrix subcells; maximum versus minimum modulus microstructures. Dotted lines are PDFs of the strains in the minimum modulus microstructures, solid lines are PDFs of the strains in the maximum modulus microstructures. 


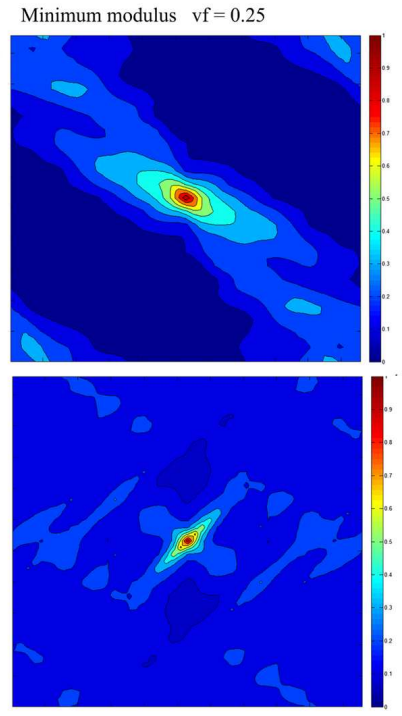

Maximum modulus vf $=0.25$
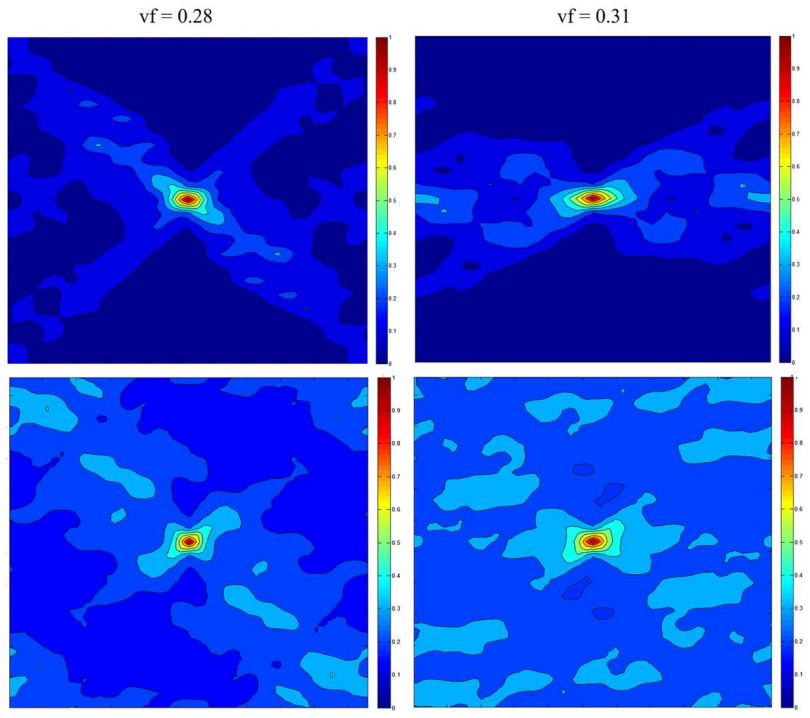

$\mathrm{vf}=0.28$

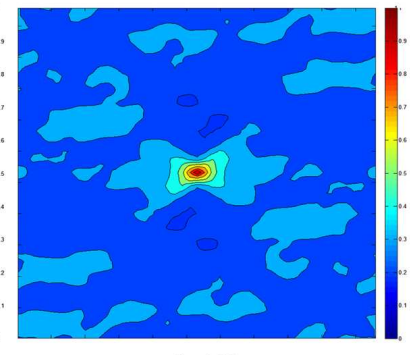

$\mathrm{vf}=0.31$

Figure 8: Autocorrelation plots of minimum modulus microstructures (top row) and maximum modulus microstructures (bottom row). Scale colors are the same for both sets of plots and correlation values are scaled from 0 to 1. 
A random spatial arrangement will be uncorrelated. Clustering, patterned spatial effects and gradients will result in higher correlation values. Figure 6 shows autocorrelation plots for volume fraction $0.25,0.28$ and 0.31 . The top row are minimum modulus microstructures, the bottom row, maximum.

The $x-y$ axes in each graph measure separation distances; the origin $C(0,0)$ is at the center of each plot. The correlation distances span \pm 24 subcells. The highest autocorrelation is always of a point with itself, which is at the origin. A wider ring of higher correlation indicates that neighboring points have similar features, in this case strains. The patterning in the plots may correspond to gradients in the strain fields. Discontinuous regions indicate regularly spaced patterns.

Both sets display patterning in the correlation values, suggesting that the strain fields are not random. The maximum modulus microstructures display slightly higher correlation values than the minimum microstructures; light blue versus darker blue. Qualitatively, both sets of plots suggest that strains can be highly correlated as far as $\sim 4-6$ subcells away. In some cases a lower degree of correlation exists at even larger distances of $\sim 10$ subcells.

\section{Summary and Conclusions}

Enhanced mechanical properties have been experimentally observed in polymer nanocomposites at uncharacteristically low volume fractions. Mathematical models based solely on the percolation theory of connected microstructures include neither the effects of the formation of an interface region between the matrix and included phase, which is significant at the nanoscale, 
nor the influence of complex microstructures on the mechanical response of the composite.

A simulation model using the GMC computational micromechanics model was used to predict the properties due to random microstructured nanocomposites. This modeling approach included both the effect of irregular random spatial positioning of the particle phase, e.g., long and short range connectivity and/or clustering, and the effect of the micromechanical contribution of an interface region with stiffer properties than the matrix. Model predictions corresponded well to observed experimental results. While the most significant increases in stiffness appeared due to obviously connected microstructures, the observed distribution of properties, resulting from 'not obviously' connected microstructures is more likely due to combined microstructural and micromechanical effects.

The role of composite micromechanics, using the very narrow definition of the degree to which the volume fraction and stiffness of composite phases contribute to the effective properties, was illustrated by using the classic Mori-Tanaka micromechanics model to predict the properties of the three phase composite. MT captures the influence of the three separate phases, but includes only indirect effects of microstructure. The MT model results predicted a significant, but gradual, increase in composite stiffness with increasing volume fraction.

Results suggest that both microstructure and micromechanics contribute to the observed material response; microstructural effects appear to produce the largest increases in stiffness; micromechanical mechanisms have a more significant influence on the distributions of properties. 
To further investigate these combined microstructural/micromechanical effects, a novel approach was taken where the evolution of strain fields associated with random microstructures, rather than the microstructures themselves, were tracked in a probabilistic framework. These strain fields were calculated for random microstructures developed in the GMC/Simulation model of a 2D, 3-phase nanocomposites and provided a clear visual link between particle placement, microstructure, and a mechanical mechanism, strain. Trends in these strain fields were characterized in two ways. First, probability density functions of the strains were constructed using the Principle of Maximum Entropy, and second, spatial autocorrelation fields were calculated for the strain fields of selected microstructures.

The statistical analysis of the strain fields suggests several different types of mechanical mechanisms.

1. At low volume fractions it is likely that a significant portion of the increase in stiffness is due to the addition of a third phase with greater stiffness than the matrix. Regions of interface are concentrated around the particles, and interface-particle connections keep interface strains small.

2. As particle volume fraction increases, the distribution of elastic strains in all of the phases becomes relatively symmetric and the range of matrix strains narrows. This suggests that fewer regions of the matrix are isolated from regions of interface or particles and so they are more uniformly reinforced by the stiffer phases. In this range microstructure plays a more significant role, heavily influencing, decreasing, the load that the matrix material carries. 
3. In the range where the volume fractions of particles and interface are approximately equal, the matrix material plays less of a role in the composite properties and the composite mechanics of a two phase material, particles and interface, dominates.

Understanding the mechanical mechanisms that produce a percolationlike effect in nanocomposites requires the development of predictive micromechanical models and analysis techniques that include parameters linked to specific mechanics-based mechanisms. In particular, models must reflect the properties and geometry of interfacial regions and provide a method for analysis that can capture the underlying non-deterministic mechanisms of random spatial positioning of the reinforcing phase. This work demonstrates one such modeling framework that can be used to predict low volume fraction percolation effects. The analysis suggests that the effective properties of the composite are likely the result of overlapping qand interacting mechanisms; the influence on the composite of a significant volume fractions of a stiff interface, the effective replacement of matrix by interface, and the contribution of the interface to the formation of a reinforcing/supporting, potentially connected, microstructure.

\section{Acknowledgments}

This work was supported by the National Science Foundation CMMI: \#1030162. We would like to thank NASA-Glenn, Dr. Steve Arnold, for access to the GMC-MAC software. 


\section{References}

[1] A. Celzard, E. McRae, C. Deleuze, M. Dufort, G. Furdin, and J.F. Mâreché. Critical concentration in percolating systems containing a high-aspect-ratio-filler. Physical Review B, 53(1):6209-6214, 1996.

[2] V. Favier, H. Chanzy, and J-Y. Cavaillé. Polymer nanocomposites reinforced by cellulose whiskers. Macromolecules, 28:6365-6367, 1995.

[3] M. Niklaus and H.R. Shea. Electrical conductivity and young's modulus of flexible nanocomposites made by metal-ion implantation of polydimethylsiloxane: The relationship between nanostructure and macroscopic properties. Acta Mater., 59:830-840, 2011.

[4] R. Qiao and L.C. Brinson. Simulation of interphase percolation and gradients in polymer nanocomposites. Composite Science and technology, 69:491-499, 2009.

[5] R.V. Kurahatti, A.O. Surendranathan, S.A. Kori, and N. Singh. Defence applications of polymer nanocomposites. Defence Science Journal, $60(5): 551-563,2010$.

[6] H.W. Wang, H.W. Zhou, R.D. Peng, and L. Mishnaevsky Jr. Nanoreinforced polymer composites: 3D FEM modeling with effective interface concept. Composites Science and Technology, 71:980-988, 2011.

[7] R.A. Vaia and J.F. Maguire. Polymer nanocomposites with prescribed morphology; going beyond nanoparticle-filled polymers. Chemical Materials, 19:2736-2751, 2007. 
[8] S.C. Baxter and C.T. Robinson. Pseudo-percolation: Critical volume fractions and mechanical percolation in polymer nanocomposites. Composites Science and Technology, 71:1273-1279, 2011.

[9] B.S. Fralick, E.P. Gatzke, and S.C. Baxter. Three-dimensional evolution of mechanical percolation in nanocomposites with random microstructures. Probabilistic Engineering Mechanics, 30:1-8, 2012.

[10] D.S. McLachlan, M. Blaszkiewicz, and R.E. Newnham. Electricalresistivity of composites. Journal of the American Cermaic Society, 73:2178, 1990 .

[11] N. Ouali, J-Y. Cavaillé, and J. Perez. Elastic viscoelastic and plastic behavior of multiphase polymer blends. Plastics, Rubber and Composites Processing and Application, 16:55-60, 1991.

[12] G. D. Seidel and D. C. Lagoudas. A micromechanics model for the electrical conductivity of nanotube-polymer nanocomposites. Journal of Composite Materials, 43(9):917-941, 2009.

[13] H. Liu and L.C. Brinson. Reinforcing efficiency of nanoparticles: A simple comparison for polymer nanocomposites. Composites Science and Technology, 68:147-162, 2007.

[14] R. Bourn, B.S. Fralick, and S.C. Baxter. Distributions of elastic moduli in mechanically percolating composites. Probabilistic Engineering Mechanics, 34:67-72, 2013.

[15] M. Paley and J. Aboudi. Micromechanical analysis of composites by the generalized cells models. Mechanics of Materials, 14:127-139, 1992. 
[16] J. Aboudi, S.M Arnold, and B.A. Bednarcyk. Micromechanics of Composite Materials: A Generalized Multiscale Analysis Approach. Butterworth-Heinemann/Elsevier, 2013.

[17] T. Mori and K. Tanaka. Average stress in matrix and average elastic energy of materials with mis-fitting inclusions. Acta Mettallurgica, 2:571-574, 1973.

[18] Y. Benveniste. A new approach to the application of Mori-Tanaka theory in composite materials. Mechanics of Materials, 6:147-157, 1987.

[19] T. Mura. Micromechanics of Defects in Solids. Martinus Nijhoff/ Kluwer Academic Publishers Group, 1987.

[20] S. Torquato. Random Heterogeneous Materials: Microstructure and Macroscopic Properties. Springer, New York, 2001.

[21] B. D. Ripley. Spatial Statistics. Wiley-Interscience, 1981.

[22] E. T. Jaynes. Information theory and statistical mechanics. Physics Review, 106:620-630, 1957.

[23] E.T. Jaynes. Probability Theory: the Logic of Sciences. Cambridge University Press, 2003.

[24] Stevens D. R., E. W. Skau, L. N. Downen, M.P. Roman, and L.I. Clarke. Finite-size effects in nanocomposite thin films and fibers. Physics Review E, 84:021126-1-021126-5, 2011. 
[25] A.I. Beltzer and T. Satu. Probability distribution of wave velocity in heterogeneous media due to random phase configuration. Wave Motion, 38:221-227, 2003. 